\title{
Italique
}

Poésie italienne de la Renaissance

XV | 2012

Varia

\section{«Ecco mormorar l'onde». Un esercizio di lettura}

\section{Salvatore Ritrovato}

\section{OpenEdition}

\section{Journals}

Edizione digitale

URL: https://journals.openedition.org/italique/356

DOI: 10.4000/italique.356

ISSN: 1663-4438

\section{Editore}

Librairie Droz

\section{Edizione cartacea}

Data di pubblicazione: 1 décembre 2012

Paginazione: 195-214

ISBN: 978-2-600-01639-1

ISSN: 1423-3983

\section{Notizia bibliografica digitale}

Salvatore Ritrovato, ««Ecco mormorar I'onde». Un esercizio di lettura», Italique [Online], XV | 2012, online dal 01 décembre 2015, consultato il 21 septembre 2021. URL: http://journals.openedition.org/ italique/356 ; DOI: https://doi.org/10.4000/italique.356 
SALVATORE Ritrovato

«E C C O M O R M O R A R L'O N D E».

UN ESERCIZIO D I LETTURA 



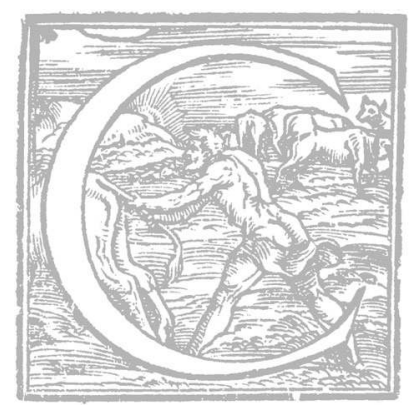

he cosa si può aggiungere di nuovo alla fine lettura che Antonio Daniele, ormai nel 1972, diede di Ecco mormorar l'onde?' Daniele si soffermava in modo particolare sul tema dell'alba e sulle sue implicazioni poetiche, fornendo interessanti spunti di riflessione sulla lingua del testo, che nella rarefazione, persino un $p o^{\prime}$ leziosa, degli elementi lirici ( $E$ soura $i$ verdi rami i vaghi augelli») comporta tuttavia una «superiore sintesi musicale»; infine entrava nel merito dello schema metrico del madrigale, sottolineando la forza strutturante del settenario (anche nel ritmo degli endecasillabi), e dell'«ordinamento quasi matematico» dei versi raggruppabili in due cola iniziali simmetricamente disposti su sei e cinque versi ciascuno, in rima baciata. Brevemente accennava Daniele al rapporto con la ballata Ecco sparir le stelle e spirar l'aura, senza entrare nel merito della loro cronologia, e, alla fine del saggio, richiamava l'attenzione sul madrigale 'gemello' Ore, fermate il volo. Il rischio di ripetere quanto già detto è alto, e giustamente Massimiliano Chiamenti, in una Glossa di accompagnamento per un madrigale tassiano apparsa sul sito di "Nuovo Rinascimento» nel settembre del 1997, ${ }^{2}$ si chiedeva se non era il caso di tacere davanti a questo esile e felicissimo madrigale. Salvo puntualizare con profitto alcune scelte lessicali, come i verbi parasintetici (di suggestione dantesca) imperlare $e$ indorare, da Tasso definiti, nei Discorsi del poema eroico, "fatti》 o "finti»,3 iquali concorrono al "largo" degli ultimi versi, e di altri "imitativi" come mormorar, che, osserva il poeta, portano seco, agli occhi e alle orecchie dei lettori, l"«evidenza») della poesia. ${ }^{4}$

La lettura di Daniele, di là dai suoi meriti interpretativi, resta esemplare anche sul piano metodologico, dal momento che non si propone di intendere il madrigale nel suo significato letterale, cosi da giungere alla parafrasi (penso al modello di "esercizio di lettura" che Gianfranco Contini fece su Tanto gentile e tanto onesta pare) come a un punto fermo della complessa esegesi testuale. No, si può dire che il madrigale (ove non si parli ovviamente di quelli di Michelangelo) non abbia bisogno tanto di parafrasi, quanto di ascolto. Ed è su questo piano che è possibile ora sviluppare dai margini del lavoro di Daniele nuove prospettive interpretative intorno a questo madrigale tassiano. 
Ecco mormorar l'onde

e tremolar le fronde

a l'aura mattutina e gli arboscelli,

e sovra i verdi rami i vaghi augelli

cantar soavemente

e rider l'oriente;

ecco già l'alba appare

e si specchia nel mare,

e rasserena il cielo

e le campagne imperla il dolce gelo,

e gli alti monti indora.

O bella e vaga Aurora,

l'aura è tua messaggera, e tu de l'aura

ch'ogni arso cor ristaura.

Ecco mormorar l'onde (n. I43) '̀ una lievissima rappresentazione di elementi naturali colti in rapida, fugace successione, fino alla comparsa in scena della donna, evocata mediante il senhal petrarchesco de "L'aura» (cui non è estranea, suggerisce lo stesso Tasso, un luogo dantesco), ${ }^{6}$ e implicata in un gioco paronomastico di rime e rispondenze

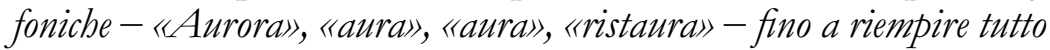
il componimento delle sue liquide, / $\mathrm{r} / \mathrm{ed} / \mathrm{l} /$. Gli elementi naturali trascorrono velocemente fondendosi in un quadro unitario e coerente, assecondato sia dalla struttura sintattica (che si svolge in due periodi: il primo, vv. I-II, suddiviso in due membri introdotti da «ecco», costituiti da una serie di frasi coordinate dall'infinito, che produce rime e assonanze interne, e da parallelismi e chiasmi, alternati e sourappostiparall.: «mormorar l'onde» vs «tremolar le fronde», ch. «tremolar le fronde / a l'aura» «soura i rami angelli / cantar», parall. «cantare soavemente» «rider l'oriente», ch. «rasserena il cielo» «le campagne imperla», «imperla il dolce gelo» "gli alti monti indora»-; il secondo, vv. I2-I4, che forma una sorta di 'congedo' da canzone, che ripete quattro volte aura, in rima ed epanadiplosi) sia dalla struttura metrica (con netta prevalenza di "versi rotti", e sequenza di rime baciate e a eco, all'inizio e alla fine del testo, vv. I-2 e I3-I4).

Incluso, insieme a un altro celebre madrigale (n. I44),

Ore, fermate il volo

nel lucido oriente, 


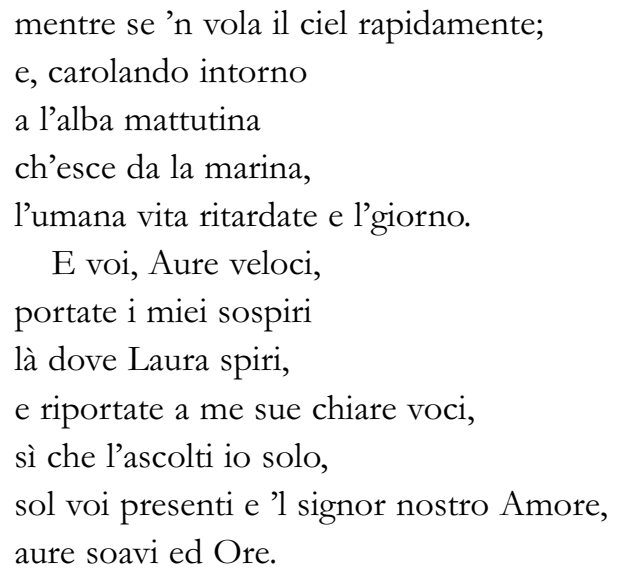

in un canzoniere di rime per Laura Peperara, conosciuta nel I563, esso appartiene alla giovinezza del poeta, e si puo dire che rappresenti uno dei risultati più brillanti e convincenti della lirica madrigalesca del Tasso e di tutto il Cinquecento. A questo canzoniere, che per comodità viene suddiviso in "innamoramento" (testi nn. I30-I56, cbe contengono una sequenza di componimenti sull'aura, I32-I46), "gelosia" (nn. Is7I76) e "corteggiamento" (nn. I77-204), il poeta cominciò a scrivere nel I563, e vi terminò nel I583. Ma nel I583 l'edizione Vasalini delle Rime, et prose tassiane, ci consegna una versione rimaneggiata, in forma di ballata, ${ }^{7}$ dei due madrigali in questione, Ecco sparir le stelle e spirar l'aura $e$ Ore fermate il volo: ${ }^{8}$

Ecco sparir le stelle e spirar l'aura, e tremolar le fronde

de gli arboscelli al mormorio de l'onde, che 'l suo spirto dolcissimo ristaura,

e tra' frondosi rami i vaghi augelli cantar soavemente; e già l'aurora ne l'oriente rugiadosa appare e le campagne imperla e i monti indora, e dispiegando al vento $i$ bei capelli chiaro specchio si fa de l'ampio mare.

$O$ bella aurora, al cui venir più care sono tutte le cose, più liete, più ridenti e più gioiose, l'aura è tua messaggiera e tu di Laura. 
Hore, fermate il volo ne l'Oriente, hor ch'un bel dì vien fore con sì lieto splendore.

E carolando intorno a l'Alba matutina ch'esce de la marina, la nostra vita ritardate e 'l giorno, il qual nel suo ritorno, benché più bel rinasca o ver maggiore, non la rende a chi more.

Aure veloci, e voi portate i miei sospiri là dove Laura spiri, e riportate i chiari accenti suoi ov'io gli ascolti poi sol voi presenti e 'l Signor nostro Amore, Aure soavi et ore.

Versioni che Tasso accoglie all'interno di un nuovo corpus di rime conservato nel manoscritto Chigiano L VIII 302, esamplato dall'autore a S. Anna intorno al I584,' e quindi nell'edizione autorizzata Osanna, dove però sono classificate, a dispetto della forma, come "madrigali". Per quanto le ballate compaiano solo in un manoscritto del I584, non si può dire che esse siano state scritte dopo i madrigali. Ma vale il contrario. Se l'esame del corpus codicologico stabilisce, in apparenza, una cronologia certa, l'ideale storia stilistica del Tasso non offre alcuna certezza. Le ballate potrebbero essere un rimaneggiamento dei madrigali della giovinezza, forse in vista di una raccolta organica di rime (penso a Delle rime del S. Torquato Tasso Parte Quarta e Quinta, Orero, Genova I586). Quando Claudio Monteverdi fornisce una superba interpretazione musicale di Ecco mormorar l'onde (Secondo libro de madrigali, Vincenti, Venezia I590), Tasso torna ai "madrigali" inserendoli, con un lavoro rigoroso e coerente di selezione, nella nuova raccolta Osanna (Delle Rime del sig. Tor-

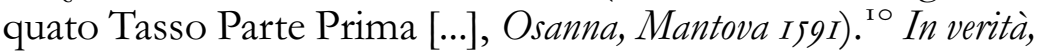
non saprei dire quanto conti la precedenza cronologica ai fini di questo contributo, se non per mettere in rilievo (ammessa la seriorità delle ballate) come la cristallina bellezza di Ecco mormorar l'onde, testo che nasce all'altezza dei Discorsi dell'arte poetica, ha qualcosa di 
davvero singolare. Singolare non solo in rapporto a Ore, fermate il volo $o$ alle ballate sopra citate, ma anche nell'ambito della forma del madrigale cinquecentesco.

Si può dire che i quattro testi, stesi a distanza di anni fra loro, circoscrivano la medesima isotopia tematica del sorgere dell'alba come "correlativo oggettivo" di un'insorgenza amorosa, che ritrova nella scenografia naturalistica (dal cantare degli uccelli alla danza delle ore, dal mare che illimpidisce al mare che culla l'alba, fino all'ampio trascolorare di un cielo d'«oriente», fino alla brezza ristoratrice) la facoltà mimetica propria del madrigale, inteso - avverte Strozzi il Giovane nella Lezione sopra il madrigale (I574) - a imitare anche azioni non umane:

Ma perché in tutti i madrigali non è azione umana, bisogna ricordarsi di quello che si disse di sopra, dove e' si mostrò che e' conveniva al poeta dare azione a tutte le cose; e così non si verranno a escludere quegli altri che [non] contengono operazione umana e sono nondimeno poesie molto vaghe, sì come sono alcuni che altro non fanno che descrivere l'apparire dell'aurora, percioché la vaghezza loro in questo consiste, che e' non ne parlano in quella guisa che farebbe uno astronomo o un filosofante, ma dicono che ella apre con la sua mano argentata la porta d'Oriente e che ella spiega lassù un velo vermiglio e sparge su l'erba delle perle di rugiada, e mille altre azioni sì fatte attribuendo le vanno. ${ }^{11}$

E ragionevole pensare che lo Strozzi si riferisse ad altri autori che trattano un topos attiguo a quello tassiano, da non confondere, qual è la donna che sorge dal letto come una luminosa aurora. Per esempio, Anton Francesco Rainerio, in un sonetto, mette l'uno di fronte all'altro l'aurora e l'amata, dopo aver descritto - non senza una punta di elegante, parnassiano compiacimento - l'epifania dell'alba:

Era il mar cheto, e l'alte selve e i prati scoprian le pompe sue, fior, frondi al cielo, e la notte se 'n gìa, squarciando il velo e spronando i destrier foschi et alati.

Scotea l'Aurora da' capegli aurati perle d'un vivo trasparente gielo; già rotava la luce alma di Delo raggi da i liti Eoi ricchi odorati. 
Quand'ecco d'occidente un più bel sole spuntogli incontro, serenando il giorno, e impallidìo l'orientale imago.

Velocissime luci eterne e sole, con vostra pace, il mio bel viso adorno parve allor più di voi lucente e vago. ${ }^{\mathrm{I}}$

Alla prima quartina incentrata sulla metamorfosi atmosferica, segue una seconda animata da una certa "altezza di stile», per cui si è anche parlato di classicismo petrarchista (dietro il ricco tendaggio dei riferimenti mitologici, si nota l'imitatio di un epigramma di Quinto Lutazio Catulo) ${ }^{{ }^{3}}$ e se $i$ «capegli aurati» rimandano alla ballata tassiana, «serenando il giorno» ci riporta per un attimo al madrigale. Quel che manca, in questo componimento, di genere più descrittivo che evocativo, è la dolce e lieve brezza della mattina. ${ }^{14}$ La brezza che innesca, in quanto aura-mot, passata a suo tempo dalla lirica romanza al poema dantesco, al canzoniere petrarchesco - in cui si affaccia fra il tripudio mattutino della natura, in Il cantar novo e 'l pianger delli augelli (Rvf 219), epifania sincretica di Laura / aurora ("1...Ivi è Laura ora»), in Quand'io veggio dal ciel scender l'aurora ( $\operatorname{Rvf} 29 I) e$ l'amarcord alle acque di Valchiusa, e in Sento l'aura anticha, e i dolci colli $(\operatorname{Rvf} 320)^{15}$ - l'aura-situation dei testi tassiani: qui l'aura, l'alloro e l'aurora concorrono a sostenere, entro una cornice di felice idillio, l'identificazione e la reversibilità (messa in evidenza in un passaggio centrale del testo: "Ecco già l'alba appare / e si specchia nel mare») fra la donna e la natura, dove «la natura non è più solo sfondo cortigiano alle bellezze della figura femminile, ma assorbe quella stessa figura e ne manifesta i trattì. ${ }^{6}$ Qui, possiamo aggiungere, il 'mezzo' prescelto, cioè il metro del madrigale, diventa 'messaggio' della poesia, della sua musicale leggerezza.

Occorre, dunque, partire dalla forma. Nei I4 versi che compongono $i$ due madrigali e la ballata grande (composta di ripresa XyYX, due mutazioni $A B C / B A C$, e volta $C d D X)$, e nei I7 della ballata mezzana (suddivisa in ripresa $\times Y y$, e due stanze, composte di due mutazioni $a b / b A$, e volta a $Y$ ), si iscrivono come su un diagramma analogie e differenze. Mentre Ore fermate il volo punta ad abbinare le rime, a regolare cadenza di rima baciata (abBcddCeffEaGg), con 
prevalenza di settenari (4 contro is endecasillabi della ballata), a sollievo dell'avvolgente trama polisindetica che sviluppa l'assimilazione finale aura-laura; Ecco mormorar l'onde presenta un tessuto metrico più semplice (sette coppie di rime baciate: a aBBccddeEffGg), appena increspato da 4 endecasillabi che la versione in ballata trasformerà in un movimento triadico di rime variamente concatenate (AbBACDEDCEEfFA), e senza dubbio più lento (gli endecasillabi prevalgono sui settenari, I2 a 2). In entrambi i madrigali, nel terzetto conclusivo, compare lo schema della ripresa, con una sequenza di metri invertita rispetto alla norma che vorrebbe il verso lungo come ultimo (che, si noti, nella copia postillata dell'edizione Vasalini, il poeta corregge con "Che m'accende e ristaura», restaurando l'endecasillabo). E in entrambi $i$ madrigali non si dà rima irrelata: chiusa, in Ecco, subito da una baciata («onde» / «fronde»), e in Ore, a distanza, da una concatenante (《volo» «solo»).

Le peculiarità più interessanti, sotto il profilo formale, riguardano Ecco mormorar l'onde. Mentre in Ore, fermate il volo blocchi di versi sono restituiti quasi intatti nelle quartine a rima incrociata ( $v v$. $5-7 m=v v .4-7 b ; v v .8-I 4 m=v v$. II-I7b); in Ecco mormorar l'onde Tasso sospinge linearmente il tracciato metrico, con brevi e concisi tagli nella trama polisindetica che riparte anaforicamente a inizio verso. Inoltre, Ore, fermate il volo affaccia ambiguamente il fantasma della ballata nella sua struttura madrigalesca, ripetendo ripresa, mutazioni e volta (quest'ultima appena variata nel suo ordine rimico d'apertura, $a b B \leftrightarrow a G g$, simulando la replica di una ripresa di ballata, con l'energica invocazione alle Ore: "Ore, fermate il volo [...] si che l'ascoltio solo»), e nel suo oscillare fra la libertà formale del madrigale e lo schema della ballata, richiama quel tipo di madrigale che Don Harrán ha chiamato "a ballata", in cui la volta non ripete necessariamente le rime della ripresa ma si l'ordine delle rime. ${ }^{17}$ Certamente, il raffinato reticolo di equipollenze formali delle ballate, in cui le strutture linguistiche sembrano continuamente slittare sul metro, raddoppiando talora il passo e creando improvvise inarcature, quasi a smentire la staticità della forma chiusa, trova nel madrigale, in una forma svincolata dal rispetto di uno schema (sia pure non rigido), un nuovo principio di armonizzazione metrico-ritmica, determinato in parte dalla prevalenza di settenari che impongono precisione alle immagini, in parte dall'ordine delle rime che, nel loro disporsi liberamente, inducono il poeta a puntare 
sulla coesione e l'agilità melodica dei versi (non a caso, una rima madrigalesca come «veloci» / «voci» scompare nella ballata in «voi» / «suoi»).

Questa medesima struttura metrica, con un leggero incremento di endecasillabi (s unità contro 4), si ravvisa in Secco è l'arbor gentile, indicato come madrigale (abBcddCeffEaGG). Qui il collaudato ordine compositivo (terzina + due quartine a rima incrociata + terzina), oltre a coincidere con la struttura sintattica del testo, si sovrappone alla sequenza dei versi, raggruppabili in due terzine e due quartine: queste, composte da settenario settenario settenario endecasillabo; quelle, di settenario settenario endecasillabo e settenario endecasillabo endecasillabo. Rime concatenanti («legge» / «regge», «oro» / «alloro», «gentile» / «aprile») creano corto circuiti all'interno del testo, scavalcando distici e terzetti:

Secco è l'arbor gentile che mai le fronde e 'l verde o per gielo o per fulmine non perde.

O mutata è la legge de la natura, o 'l sole men può di quel che suole; e sol le stelle Amore e 'l mondo regge, e col piombo e con l'oro miracoli rinnova, e fa vendetta nova d'antico oltraggio ne l'amato alloro.

Ma se nel lieto aprile rinverdir al mio crin non dee corona, secchisi anco Permesso in Elicona. ${ }^{18}$

Il concatenarsi delle rime ha l'effetto di amplificare, come in un gioco di echi e risonanze, $i$ diversi motivi che si intrecciano nel testo, stemperando eventuali rigide, per quanto effimere, simmetrie che ricordano quelle di una stanza di canzone. Altri madrigali tassiani si fondano, invece, sulla replica di terzine in ordine ascendente di settenario settenario endecasillabo, nonché sulla funzione di sutura delle rime incrociate. Questo avviene (per restare alle rime per Laura Peperara) in Messaggera de l'alba: 
Messaggera de l'alba

è quest'aura terrena

e torbida talor, talor serena:

Laura mia par celeste,

così bella io la veggio

dopo l'aurora in fresco e verde seggio:

di fior l'una riveste

il dilettoso aprile,

l'altra fiorir fa l'amoroso stile. ${ }^{\text {'9 }}$

Ovviamente la disposizione tipografica dei testi, nelle raccolte a stampa dell'epoca (ma persino nell'edizione critica, pur meritoria, del Solerti) non è probante per risalire alle differenze formali, anzi andrebbe attentamente riconsiderata e forse in una prospettiva critica che induca piu che a sottili discussioni sull'attribuzione dei generi metrici, a un'attenta riflessione sulla tensione metamorfica del metro lirico, dalla canzonetta al madrigale alla ballata. ${ }^{20}$ Raccogliendo un altro esempio dalle rime per la Peperara (n. I37):

Non fonte, fiume, od aura odo in più dolce suon di quel di Laura. né 'n lauro, o 'n pino, o mirto, mormorar s'udì mai più dolce spirto.

O felice, a cui spira, e quel beato, che per lei sospira.

Che se gli inspira il core, pote al Ciel aspirar co 'l suo valore. ${ }^{21}$

Nella visualizzazione dei rientri tipografici della stampa originaria si intuisce come la libertà del madrigale sia regolata dall'intrecciarsi e giustapporsi delle rime che occupano lo spazio del testo, senza chiuderlo (come avviene nei sonetti o nelle stanze di canzoni) in uno schema prevedibile. Il rapporto metro-lingua è tanto pervasivo nel madrigale da ipotecare una impaginazione non predittiva, in cui le convenzioni tipografico-editoriali interagiscono con quelle retorico-letterarie (parallelismi sintattici, iterazioni, anafore, antitesi, inversioni, chiasmi), e danno vita - cosi come avverrà nella poesia moderna - a una versificazione dinamica, in quanto varia da una poesia all'altra, da un autore all'altro. ${ }^{22}$ Senza dubbio, il distico e il tristico forniscono un'unità di 
misura fisiologica nel respiro del madrigale, ${ }^{23}$ ricomponendo le distorsioni metrico-sintattiche entro la soluzione monostrofica del madrigale. ${ }^{24}$ Comunque sia, autori e editori tendono a raggruppare $i$ madrigali rispettando ora la coerenza delle rime ora l'unità delle strutture linguistiche, ora l'una e l'altra. Trascriviamo un madrigale tassiano rispettando $i$ capoversi della stampa originale:

Se taccio, il duol s'avvanza;

se parlo, accresco l'ira,

Donna bella, e crudel, che mi martira.

Ma pur prendo speranza, che l'umiltà vi pieghi, che nel silenzio ancor son voci e prieghi;

e prego Amor, che spieghi

nel mio doglioso aspetto

con lettre di pietà l'occulto affetto. ${ }^{25}$

La scansione in tre terzetti incatenati fra loro $(a b B / a c C / c d D)$ rievoca la trama di una stanza di canzone, con due piedi e una sirima, e si distingue per la coerenza dei passaggi. I tre periodi metrici coincidono con una forte cesura sintattica; all'interno di ogni terzetto e fra $i$ tre terzetti è possibile intravedere un principio di equivalenze strutturali che, sottolineando con una duplice gradatio ("sse taccio», «se parlo», «ma pure spero», «e prego»: ovvero, dai «martiri» ai «prieghi» all"«occulto affetto») lo svolgimento del tema, e rigenerandosi nelle relazioni esplicative del se $e$ del che, offre una ragione decisiva al raggruppamento triadico. Altrimenti è possibile che due periodi metrici dal passo diverso (come si vede nel madrigale «d'incerto autore» sotto citato) - il primo di due quartine a rime intrecciate e fra loro concatenate $(A b B C / A D d c)$ e il secondo di tre distici baciati (EefFGG) - si sourappongano, confermando con il rientro del solo incipit e la contrapposizione fra $i$ due piedi dal passo ineguale e una sirma priva di ogni legame di rima, l'origine della stanza di canzone:

Come purpureo fior vinto dal gielo languido venir suole, poi vago aprirsi a l'apparir del sole, tal per nuovo accidente il bel colore cangiar vid'io sotto candido velo a la mia donna, e restar bianca neve, 
indi tornar in breve

lo smarrito vigore;

ed ella lieta dolcemente e bella,

al par d'ogni altra stella,

ond'io ben dissi alora:

«O felice, per cui si discolora,

e poi riprende sua beltà divina

questo vago, leggiadro fior di spina». ${ }^{26}$

I due principi formali che qui vediamo agire dinamicamente - l'osservanza di una certa distribuzione delle rime fra piedi e sirma, da un lato, e la libertà della loro disposizione con un'alternanza di endecasillabi e settenari, dall'altro - sono propri anche della stanza di canzone. Lecito domandarsi se è corretto parlare di un "madrigale a canzone", che non costituisca un gradino intermedio fra la canzone divisa e il madrigale, ma una peculiare declinazione metrica di questo genere, la cui libertà formale va interpretata come un'apertura sperimentale, non esente dall'osservanza di alcune regole (o non-regole) strategiche. ${ }^{27}$ Tanto per il madrigale a ballata, quanto per quello a canzone si accampano, come possibili provvedimenti tecnici, l'omissione di una rima nel secondo piede o della concatenatio, l'introdurione di una nuova rima fra la prima e la seconda parte che infrange la sequenza metrica, e la dislocazione asimmetrica delle rime, che ba modo ovviamente di esprimersi meglio in un disegno monostrofico. In tal senso, l'accostamento del madrigale alla canzone divisa non va inteso come genetico, di derivazione l'uno dall'altra, bensi 'metamorfico', nella misura in cui il madrigale riproduce, nella sua libertà metrica, il tipo formale e concettuale della canzone divisa, e riesce ad assimilarlo, variandolo ed eludendolo. ${ }^{28}$

Tornando a Ecco mormorar l'onde, la sua struttura sembra sfuggire a ogni facile schematizzazione e puntare invece a una linea melodica che attraversa il testo, verso per verso, evitando «rompimenti lunghì, ardite figure di inversione sintattica, torsioni metaforiche. Di pari passo procede la rastremazione lirica del topos dell'aurora, in cui il sorgere dell'alba diventa la proiezione sineddochica della presenza virtuale dell'amata. Composto, infatti, a «imitazione» di una terzina del Purgatorio dantesco, ${ }^{29}$ cui forse va aggiunto almeno un'altra sollecitazione, persino più ricca sul piano lessicale, dal Paradiso, ${ }^{30}$ questo 
madrigale incrocia in più punti la produzione poetica tassiana, non solo quella lirica, ${ }^{31}$ ma persino quella epica, ${ }^{32}$ affermando ogni volta un suo peculiare timbro elativo, in rapporto a una situazione descritta non concettualmente bensi musicalmente, in cui l'io-poetico - mai grammaticalizzato - percepisce la nebulosa femminile afferrandosi al deittico fenomenologico «ecco». A tale disincarnazione atmosferica del paesaggio danno appiglio, sul piano dello stile: a) la ridondanza dell'ornatio ("vaghi angelli», "dolce gelo», "alti monti», "bella Aurora», aura mattutina», "«verdi rami»); b) la scelta delle forme verbali allinfinito, nella prima parte, che, potenziata dall'uso strutturale del polisindeto, ${ }^{33}$ libera il momento evocativo da ogni impegno di comprensione logica, ne sospende la percezione temporale; c) infine, la sensuale partecipazione della natura, che precipita la Sehnsucht preromantica nella aequivocatio osmotica del senhal ("l'aura» / "Laura», v. I3), una sensualità che coinvolge la lingua poetica e spiega, sul piano sintagmatico, versi 'fatui' come «E sovra $i$ verdi rami $i$ vaghi angelli» (più musicale, però, di «E tra' frondosi rami $i$ vaghi augelli» della ballata) che, disinteressati al quoziente informativo degli epiteti, puntano decisamente a esaltare le trame foniche. Tale progressiva diffrazione semantica dell' aurora risulta evidente dal raffronto con la ballata, che, al setaccio delle varianti inter e intratestuali, ${ }^{34}$ cosi si presenterebbe: ${ }^{35}$

Ecco Sparir le STelle e spirar l'aura,

e tremolar le fronde

de gli arboscelli al mormorio de l'onde,

che 'I suo spirto dolcissimo ristaura,

e tra' frondosi rami i vaghi augelli

cantar soavemente; e già [ecee] l'aurora

$<$ cantar su l'alba, che la bella fronte>

ne l'oriente rugiadosa appare

$<$ coronata di rose in cielo appare >

e le campagne imperla e $\mathrm{i}$ monti indora,

$<$ e indora il monte, $>$

e dispiegando al vento i bei capelli

chiaro specchio si fa de l'ampio mare.

O bella aurora, al cui venir più care

sono tutte le cose,

più liete più ridenti e più gioiose,

l'aura è tua messaggera e tu di Laura. 
Che cosa resta, dunque, di Ecco mormorar l'onde in Ecco sparir le stelle? Proviamo a riassumere: a) il madrigale preferisce alle figure allitteranti (che nella ballata si concentrano nel ritorno periodico del nesso sordo SP/ST) il gioco limpido delle rime e delle assonanze / consonanze; b) nel diverso peso delle sillabe - I46 della ballata contro le II4 del madrigale - è inevitabile che i primi a fare le spese siano gli epiteti e le parti del discorso che introducono strutture ipotattiche, come il gerundio e il relativo; c) per quanto riguarda le immagini, nel madrigale ogni verso tende trovare una propria unità icastica, nella ballata registriamo almeno 4 inarcature forti, che conferiscono una certa gravitas al testo, e un paio di minore intensita. ${ }^{36}$

Prova decisiva del singolare valore di Ecco mormorar l'onde, a distanza di anni dalla sua composizione, è il riscontro musicale che Monteverdi diede nel suo Secondo libro de madrigali del I590, il

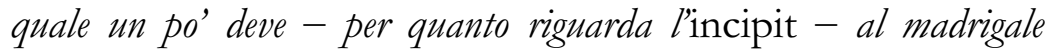
Vezzosi augelli musicato da Jaches De Wert, del I586. Com'è facile evincere dall'ascolto della composizione, a ogni verso corrisponde un periodo musicale, il quale esalta il nitore e la compiutezza metricoritmica delle immagini inscritte in ogni verso. Tramite la ripresa anaforica del deittico ecco e della congiunzione e in polisindeto, il madrigale tassiano presenta una sorta di struttura monostichica, che dissolve $i$ cola iniziali in una ripartenza continua sul ritmo del settenario, ovvero dell'endecasillabo a maiori. La «vicinità delle rime» (con parole di Bembo $)^{37}$ è un «piacevole» espediente del montaggio lirico; e non è un caso che le rime che segnano l'inizio e la fine del testo siano ad eco: in «onde» «fronde» e «aura» «ristaura», la prima parola-rima si ripercuote per intero nella seconda. ${ }^{38}$ Eे come se il madrigale fosse stato pensato, o addirittura 'versificato' (ma niente lo accerta) non secondo il canonico sistema di equivalenze a base ternaria o binaria, ma al fine di raggiungere il più alto profilo melodico, con una marcata accentuazione delle singole unità stichiche, in base alle relazioni concettuali che si instaurano allinterno della struttura poetica (parallelismi, anafore, polisindeti):

\section{a}

Ecco mormorar l'onde

b

E tremolar le fronde 
c

A l'aura mattutina e gli arboscelli,

d

E sovra i verdi rami i vaghi augelli

e

Cantar soavemente

f

E rider l'oriente;

g

Ecco già l'alba appare

h

E si specchia nel mare,

i

E rasserena il cielo

k

E le campagne imperla il dolce gelo, 1

E gli alti monti indora.

m

O bella e vaga Aurora,

n

L'aura è tua messaggera, e tu de l'aura

o

Ch’ogni arso cor ristaura.

Un capitolo non trascurabile della fortuna di questo madrigale, intonato altre cinque volte fra Cinque e Seicento, compresa la trascrizione spirituale nella raccolta del Selva; ma anche una prova convincente della sua "anima" musicale, tutta da ascoltare. ${ }^{39}$

Salvatore Ritrovato 
I. A. Daniele, Lettura di un madrigale tassesco, in «Giornale Storico della Letteratura Italiana», CXLIX, fasc. 466-67 (1972), pp. 349-62.

2. M. Chiamenti, Glossa di accompagnamento per un madrigale tassiano, 3 settembre 1997, in www.nuovorinascimento.org.

3. Torquato Tasso, Discorsi dell'arte poetica e del poema eroico, a cura di L. Poma, Laterza, Bari i 964 , p. i 80.

4. Tasso, Discorsi dell'arte poetica cit., p. 245.

5. Torquato Tasso, Le rime, edizione critica su i manoscritti e le antiche stampe, a cura di A. Solerti, 4 voll., Commissione per i testi di lingua, RomagnoliDall'Acqua, Bologna i 898-igo2 (nuova edizione a cura di B. Basile, Salerno Editrice, Roma i 994, vol. I, p. I 54 ).

6. «E quale, annunciatrice de li albori, / l'aura di maggio movesi e olezza, / tutta impregnata da l'erba e da' fiori» Dante, Purg. 24, I45-50, è il passo che Tasso ricorda in una nota ad locum, come spunto alla composizione ("Imitazione di Dante il quale disse: E l'aura annunciatrice de gli albori. Ma il poeta, chiamando l'aurora messaggera de la sua donna, ha risguardo non solo al tempo del levarsi ma a la bellezza de la sua donna...», Le opere di Torquato Tasso con le controversie sopra la Gerusalemme Liberata, Monti, Venezia, I739, vol. io). Ma è altrettanto possibile una reminiscenza del Cantico dei Cantici VI, 9: «ista quae progreditur quasi aurora consurgens».

7. In particolare si tratta, secondo la terminologia del tempo, di «ballate non vestite», in quanto di una sola stanza: cfr. Pietro Bembo, Prose della volgar lingua. Gli Asolani. Rime, a cura di C. Dionisotti, UTET, Torino i 966, p. I 53 ; ma si veda anche Torquato Tasso, La Cavaletta, in Dialoghi, edizione critica a cura di E. Raimondi, Sansoni, Firenze I958, v. II, t. II, p. 637.

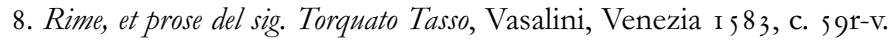

9. Torquato Tasso, Rime d'amore (secondo il Chigiano L VIII 302), a cura di F. Gavazzeni, M. Leva, V. Martignone, introduzione di V. Martignone, Panini, Modena I 993, nn. XCIII e XCIV. Secondo Raffaele Spongano (Per la futura edirione critica delle 'Rime' di T. Tasso, «Convivium», I 948, pp. 205-I 6; poi in La prosa di Galileo e altri scritti, D’Anna, Messina-Firenze I949, pp. 73-92, 2 I 2-I 3 ) il ms. si deve far risalire all'ottobre i 584 .

ıo. Si veda A. Martini, Amore esce dal caos. L'organizzazione tematico-narrativa delle rime amorose del Tasso, in «Filologia e critica», IX (1984), pp. 78-I $2 \mathrm{I}$, in part. 103 ss.

i i. G. B. Strozzi il Giovane, Lezione sopra i madrigali recitata l'anno is74 nell'Accademia Fiorentina, in Orazioni ed altre prose, Grignani, Roma I635, pp. I 59-1 8.

I 2. Lirica rinascimentale, intr. di J. Risset, a cura di R. Gigliucci, Istituto Poligrafico Zecca dello Stato, Roma 2000, pp. 680-8 I.

I 3. "Constiteram exorientem Auroram forte salutans, / cum subito a laeva Roscius exoritur. / Pace mihi liceat, caelestes, dicere vestra: / mortalis visus pulchrior esse deo» (citato da Cicerone, De natura deorum, I 79). 
I4. Come manca in un'altra possibile fonte della rima baciata finale «aura»/《ristaura»: «del suo dolce parlar lo spirto e l'aura / subitamente ogni mio mal restaura» $\mathrm{B}$ EMBo, A quai sembianza Amor Madonna agguaglia, n. LXXIII, in Prose della volgar lingua cit., p. 569 .

I 5. Un puntuale regesto di rimandi lessicali, fra Dante e Petrarca, si legge in Chiamenti, Una glossa cit.

I6. Martini, Amore esce dal caos cit., p. ı०4.

I7. D. Harràn, Tipologie metriche e formali del madrigale ai suoi esordi, in Il madrigale fra Cinque e Seicento, a cura di P. Fabbri, Il Mulino, Bologna I988, pp. 95-I 22, IO2 ss.

I 8. TAsso, Le rime cit., n. I 89 .

19. TASso, Le rime cit., n. I39.

20. Si veda a proposito A. Daniele, Teoria e prassi del madrigale libero del Cinquecento, in Andrea Gabrieli e il suo tempo, Olschki, Firenze I987, pp. 95-102; e Id., La metrica del Tasso lirico, in Linguaggi e metri del Cinquecento, Marra, Rovito I994, pp. 247-3 I 5 , in part. $260 \mathrm{ss}$.

2 I. Torquato Tasso, Delle rime, parte e seconda prima. Di novo dal medesimo in questa nuova impressione ordinate, corrette, accresciute, \& date in luce. Con l'espositione dello stesso Autore, Marchetti, Brescia I 592, p. 259.

22. Si veda ancora, a proposito, N. Ruwet, Sui rapporti fra la disposizione tipografica e la struttura linguistica in poesia, in Linguistica e poetica, Il Mulino, Bologna I986, pp. 79-101, 91 ss.

23. E questo è chiaro, oltre ogni ragionevole dubbio, già nella «forma a base ternaria» del madrigale trecentesco (cfr. P. G. Beltrami, La metrica italiana, Il Mulino, Bologna i99I, p. 28I).

24. Si veda, a proposito, il madrigale tassiano, n. 399, O vaga tortorella (sul quale è intervenuto A. Daniele, Capitoli tassiani, Padova, Antenore, I983, pp. 48-84). Un esempio di madrigale addirittura in terza rima è Se mai cortese fusti dell'Ariosto (citato da P.G. Beltrami, La metrica italiana, Bologna, Il Mulino, I991, p. 323).

25. Torquato Tasso, Rime, et prose, Parte terza, Vasalini, Ferrara I 589 , c. 39v.

26. Libro Quarto delle Rime di diversi eccellentiss. autori nella lingua volgare. Novamente raccolte, Giaccarello, Bologna I 55 , p. 309.

27. È un confine sottile e a volte inafferrabile che il poeta percorre fra chi reclama delle misure interne al madrigale e chi invece osserva totale autonomia. Valga ad esempio il passo finale della Lettura sopra un madriale di Cesare Simonetti di Ippolito Peruzzini, in cui è spiegata la particolare lunghezza del madrigale, i 5 versi contro i I 2 raccomandati dal Ruscelli. Peruzzini, che sa quanto Simonetti tenga a non "trasgredir le regole», e pure ritiene "troppo rigida» la prescrizione del Ruscelli, e si avvale senza riserve dell'autorità del Bembo (il quale fa «questa sorte di rimare 
libera, e di numero di versi, e di maniere di rime»), e porta a proposito altre due cagioni, una metrica (pertinente al nostro argomento) e una di carattere lirico, a sostegno del Simonetti: I) «avendo il madriale somiglianza con le stanze della canzone: come le stanze ponno esser più di dodici versi lunghe, e di quelle veggionsi che insino a i venti arrivano, così il madriale non dovrà essere sotto così stretta regola rinchiuso, e di peggior condizione, ch'esse stanze non sono»; 2) «massimamente, che proponendosi un poeta una materia dolce e amorosa da esser' ispiegata in un madrigale, non è conveniente ch'astretto da questa legge, o che taccia quel che voleva dire, o che, dicendolo, oscura e troncamente lo dica, essendo più giusto che le parole e i versi al concetto servano, che il concetto dalle parole o da i versi astretto sia» I. Peruzzini, Lettura sopra un madrigale del signor Cesare Simonetti da Fano: L'Assetato; recitata nell'illustre Academia de' Confusi, nel di xxiij di Marzo M.D.Lxxv, Bonardo, Bologna I 575, pp. 62-63.

28. D'accordo, dunque, con le osservazioni di Harrán sulla teoria di Massini, che sostiene che «il madrigale, quanto a forma poetica, altro non è che una stanza di canzone», anzi «per la proportione c'ha con la prima stanza della canzone, prende regola da quella quanto alla materia, forma, qualità, et quantità di versi» Harràn, Tipologie metriche, cit., p. I I 8 ss. Certo il madrigale ha molto da spartire con la canzone, quanto a materia, metro e ritmo, ma non perché deriva da questa, bensì perché entrambi fanno riferimento alla tradizione lirica italiana in generale.

29. Cfr. ivi, n. 6.

30. Purg. 28, 7-1 5: «Un'aura dolce, sanza mutamento / avere in sé, mi feria per la fronte / non di più colpo che soave vento; / per cui le fronde, tremolando, pronte / tutte quante piegavano a la parte / u' la prim' ombra gitta il santo monte; / non però dal loro esser dritto sparte / tanto, che li augelletti per le cime / lasciasser d'operare ogne lor arte».

31. Basti rimandare al sonetto tassiano n. i45 (L'aura, che dolci spirti e dolci odor), in cui è facile notare, di là dalla condivisione delle isotopie semantiche con il madrigale, l'abile tessitura degli epiteti, non sempre imprescindibili, ora in endiadi e dittologie sinonimiche, ora in monotone correlazioni bimembri che tolgono profondità al tema.

32. In un'ottava ambientata nel giardino di Armida, leggiamo: «Vezzosi augelli infra le verdi fronde / temprano a prova lascivette note; / mormora l'aura, e fa le foglie e l'onde / garrir che variamente ella percote. / Quando taccion gli augelli alto risponde, / quando cantan gli augei più lieve scote; / sia caso od arte, or accompagna, ed ora / alterna i versi lor la musica òra» TAsso, Gerusalemme Liberata, XVI, I 2.

33. Non a caso Tasso osservava nei Discorsi del poema eroico: «numerando molte cose, è meglio raddoppiar le congiunzioni, come ci ammonisce Demetrio Falereo, perché l'istessa congiunzione replicata dimostra un non so che d'infinito» TAsso, Discorsi dell'arte poetica cit., p. 207.

34. Mi riferisco, in particolare, alle postille autografe di Ecco sparir le stelle e spirar l'aura nella copia dell'edizione Vasalini conservata presso la Biblioteca Angelica di Roma, segn. Aut. I, 24. 
35. Per quanto riguarda i segni diacritici: il sottolineato individua le porzioni testuali della ballata presenti anche nel madrigale; il doppio sottolineato le parti presenti ma modificate; l'ondulato le parti che non compaiono nel madrigale; mentre in grassetto ho segnalato alcuni passaggi interessanti stilistici che rendono più elaborata, sul piano sintattico, la versione in ballata.

36. Si veda A. Afribo, Teoria e prassi della "gravitas" nel Cinquecento, Cesati, Firenze 200 I, p. I67 ss.

37. Bемво, Prose della volgar lingua cit., p. i 56.

38. «Nel caso in questione la rima ad eco non è che un macroscopico esempio di dilatazione fonosimbolica che accompagna l'aprirsi della canzone [...] e il suo chiudersi, come un indugio della voce prima del definitivo spegnersi del suono (si pensi per analogia alla musica del Monteverdi su questo stesso testo)» Daniele, Lettura cit., p. $36 \mathrm{I}$.

39. Fra i musicisti che se ne occuparono, ricordiamo Claudio Monteverdi, Antonio Dueto, Giovan Battista Galeno, e altri (cfr. Bibliografia della musica vocale profana pubblicata dal isoo al I700, a cura di E. Vogel, A. Einstein, F. Lesure, C. Sartori, Staderini-Minkoff, Pomezia i 977, n. ed.). 